\title{
A QUANTITATIVE METHOD OF COUPLER CAVITY TUNING AND SIMULATION
}

\author{
S. Zheng ${ }^{\dagger}$, Y. Cui, H. Chen, L. Xiao \\ Engineering Physics Department, Tsinghua University, Beijing, 100084, P. R. China
}

\section{Abstract}

Based on coupling-cavity chain equivalent circuit model, a quantitative method is developed to determining the coupling coefficient and frequency deviation of a coupler cavity. This method can simplify the tuning and simulation process of coupler cavity. As an example, we derived the analytical expression of the coupling coefficient and frequency deviation of a coupler cavity of periodic traveling wave accelerating tube. Compared with the method of R. L. Kyhl, it is consistent when coupling is correct. And much more, it can calculate the coupling coefficient and frequency deviation when coupler cavity is not matched.

\section{INTRODUCTION}

In accelerator tube microwave tuning, coupler matching is an important step. It decides how much microwave power can put into an accelerator tube and how pure of travelling wave field is in travelling wave accelerator.

In this paper, we describe a quantitative method of coupler matching, which is developed from method of $\mathrm{R}$. L. Kyhl [1] and Marie Chanudet [2]. It is useful in coupler cavity tuning and simulation.

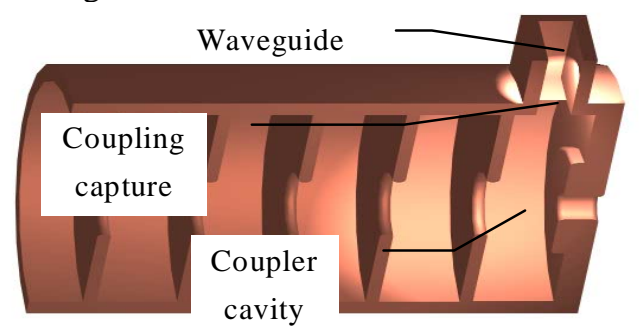

figure 1. structure of accelerating structure with a coupler

In the following section, we first describe the equivalent circuit model of monoperiodic travelling wave accelerating structure including coupler cavity. Based on this model, we derived an expression between measured reflection angle and coupler cavity frequency deviation $\Delta \mathrm{f}$ and coupling coefficient $\beta$. Then we expand this result to any travelling wave accelerating structure.

\section{MONOPERIODIC STRUCTURE}

For monoperiodic travelling accelerating structure, we repaired the equivalent circuit mode of Kyhl's. It can include the effect of coupler aperture. In this section, we assume that coupling coefficient between cavities satisfies $k<1$. So we can neglect second and higher order of $\mathrm{k}$.

zhengshuxin@263.net

\subsection{More integrated equivalent circuit model}

In order to calculating the coupling status, we must include the effect of coupling capture of coupler cavity. In repaired circuit model, a transformer is represented the field transform function of coupling capture. And because the influence of coupling capture, the equivalent inductance and capacitance of coupler cavity is not long same as others. Figure 1 shows the equivalent circuit model of Kyhl's and repaired model.
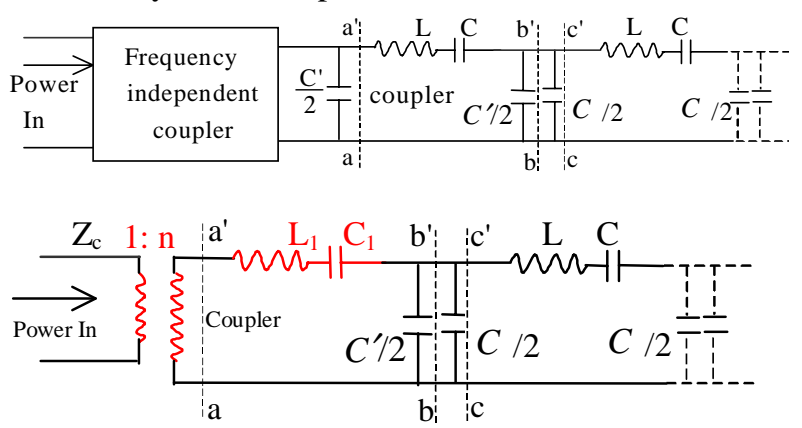

Figure 2. Equivalent circuit model of monoperiodic travelling wave structure including coupler cavity up: Kyhl's model down: repaired model

The equivalent circuit of accelerating structure is same in two models. So the following conclusion is same as reference [2]:

1. The characteristic impedance of $\pi$-series network is

$$
Z_{e}^{2}\left(\omega_{\theta}\right)=-4 X^{2}\left(\omega_{\theta}\right) \operatorname{tg}^{2} \frac{\theta}{2}
$$

Hereinto, $\theta$ is mode angle, and

$$
X\left(\omega_{\theta}\right)=\frac{1}{j \omega_{\theta} C^{\prime}}
$$

2. the $\pi / 2$ mode frequency is

$$
\omega_{\frac{\pi}{2}}=\sqrt{\frac{1}{L \gamma}}
$$

Hereinto, $\gamma$ is the series capacitance of one cavity.

$$
\frac{1}{\gamma}=\frac{1}{C}+\frac{2}{C^{\prime}}
$$

\section{2 matching parameter of coupler cavity}

Coupler matching requires impedance of accelerating structure equal to that of waveguide. At reference position a-a', the leftward impedance is 


$$
Z_{a l}=n^{2} Z_{c}
$$

and the rightward impedance is

$$
\begin{gathered}
Z_{a r}\left(\omega_{\theta}\right)=j \omega_{\theta} L_{1}+\frac{1}{j \omega_{\theta} C_{1}}+\frac{2 X \cdot 2|X| \operatorname{tg} \frac{\theta}{2}}{2 X+2|X| \operatorname{tg} \frac{\theta}{2}}= \\
|X| \sin \theta+j|X|\left(\frac{4 \delta}{k}+\cos \theta\right)
\end{gathered}
$$

Hereinto,

$$
\delta=\frac{\omega_{c}}{\omega_{\frac{\pi}{2}}}-1, \quad \omega_{c}=\sqrt{\frac{1}{L_{1} v_{1}}}, \frac{1}{v_{1}}=\frac{1}{C_{1}}+\frac{1}{C^{\prime}}
$$

Matching in operation mode $\theta_{0}$ requires that

$$
Z_{a l}=Z_{a r}
$$

so we can get

$$
\frac{n^{2} Z_{c}}{\mid X\left(\omega_{\theta_{0}}\right) \sin \theta_{0}}=\beta_{s}=1, \frac{4 \delta_{0}}{k}+\cos \theta_{0}=0
$$

and the matching frequency of coupler cavity is

$\omega_{c 0}=\omega_{\pi / 2}\left(1-\frac{k}{4} \cos \theta_{0}\right) \approx \frac{\left(\omega_{\pi / 2}+\omega_{\theta_{0}}\right)}{2}$

\subsection{Measurement step}

For tuning a coupler cavity, we must measurement the parameter at first. Figure 3 shows the measure step and corresponding circuits model.

(a)

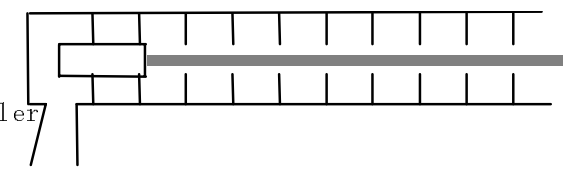

(b)
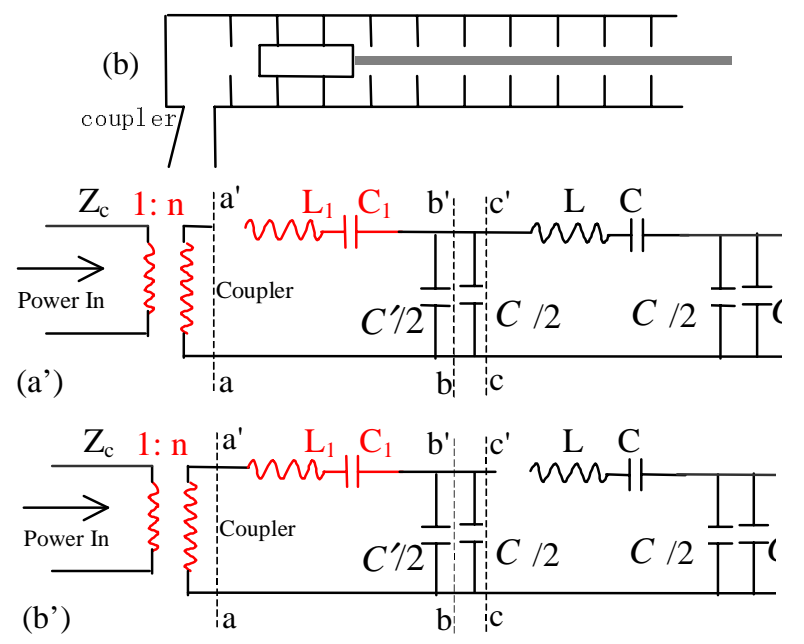

figure 3. Measurement progress and corresponding circuits model (a, a'): detuning first cavity $\left(b, b^{\prime}\right)$ : detuning second cavity

Step 1. Detuning the first coupler cavity, and measuring the reflected angles of 2 frequencies near the $\pi / 2$ mode frequency.
Step 2. Detuning the second cavity, then measuring the reflected angles of aforesaid 2 frequencies. According to the reflected angles, we can determine the matching status of coupler cavity.

\section{4 calculation of $\Delta \omega_{c}$ and $\beta$}

According to figure 3 , we can find the equation about the reflect angle $\varphi$ and any angle frequency $\omega$

$$
\operatorname{tg} \frac{\varphi}{2}=\frac{n^{2} \omega z_{c}}{L_{1}\left(\omega^{2}-\omega_{c}^{2}\right)}
$$

If we measure two reflect angles $\varphi_{1}, \varphi_{2}$ under two frequencies $\omega_{1} \omega_{2}$, we can get

$$
\begin{gathered}
\omega_{c}=\sqrt{\omega_{1} \omega_{2}} \sqrt{\frac{\operatorname{tg} \frac{\varphi_{2}}{2} \omega_{2}-\operatorname{tg} \frac{\varphi_{1}}{2} \omega_{1}}{\omega_{1} \operatorname{tg} \frac{\varphi_{2}}{2}-\omega_{2} \operatorname{tg} \frac{\varphi_{1}}{2}}} \\
\beta=\frac{1}{\frac{k}{2} \omega_{\pi / 2} \sin \theta_{0}} \cdot \frac{\operatorname{tg} \frac{\varphi_{1}}{2} \operatorname{tg} \frac{\varphi_{2}}{2}\left(\omega_{1}^{2}-\omega_{2}^{2}\right)}{\operatorname{tg} \frac{\varphi_{2}}{2} \omega_{1}-\operatorname{tg} \frac{\varphi_{1}}{2} \omega_{2}}
\end{gathered}
$$

So we can get

$$
\Delta \omega_{c}=\omega_{c}-\omega_{c 0}
$$

\section{GENERALIZE TO NONUNIFORM STRUCTURE}

According to the analyse of section 2, we can see that the tuning process is just calculating and measure the parameter of coupler cavity. Now we generalize it to nonuniform structure, and calculate and measure the frequency $\omega_{c}$ and external quality factor $Q_{c c}$ of coupler cavity.

\section{1 calculation of $\omega_{c 0}$ and $Q_{e c 0}$}

For uniform structure, we can get the analyse equation of matching frequency $\omega_{c 0}$ and external quality factor $Q_{\mathrm{cc} 0}$ of coupler cavity based on section 2 .

$$
\omega_{c 0} \approx \frac{\omega_{\pi / 2}+\omega_{\theta_{0}}}{2}, Q_{c e 0} \approx \frac{2}{k \sin \theta_{0}}
$$

For nonuniform structure, we also can get numerical result by means of numerical simulation.

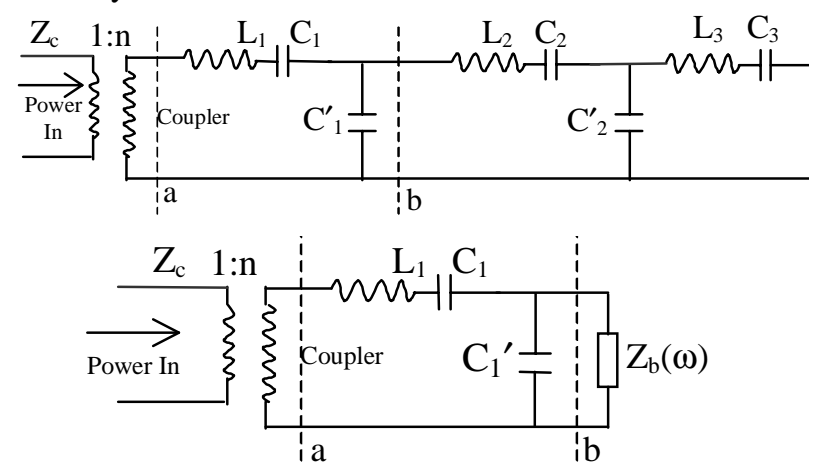

Figure 4. Equivalent circuit model of cavity-chain 


\section{2 measure of $\omega_{c}$ and $Q_{e c}$}

If the second cavity is detuned, the reflection $\Gamma$ is

$$
\Gamma=\frac{j \frac{\omega}{\omega_{c} Q_{0}}+1-\left(\frac{\omega}{\omega_{c}}\right)^{2}-j \frac{\omega}{\omega_{c} Q_{e c}}}{j \frac{\omega}{\omega_{c} Q_{0}}+1-\left(\frac{\omega}{\omega_{c}}\right)^{2}+j \frac{\omega}{\omega_{c} Q_{e c}}}
$$

So we can find $\omega_{\mathrm{c}}$ and $\mathrm{Q}_{\mathrm{cc}}$ just taking two measurements When $\mathrm{Q}_{0}$ approaching infinite, we can get $\omega_{\mathrm{c}}$ and $\mathrm{Q}_{\mathrm{cc}}$ by measure 2 reflect angles under 2 frequencies.

$$
\begin{array}{r}
\omega_{c}=\sqrt{\omega_{1} \omega_{2}} \sqrt{\frac{\omega_{1} \operatorname{tg} \frac{\phi_{1}}{2}-\omega_{2} \operatorname{tg} \frac{\phi_{2}}{2}}{\omega_{2} \operatorname{tg} \frac{\phi_{1}}{2}-\omega_{1} \operatorname{tg} \frac{\phi_{2}}{2}}} \\
Q_{e c}=\frac{\sqrt{\omega_{1} \omega_{2}}}{\operatorname{tg} \frac{\phi_{1}}{2} \operatorname{tg} \frac{\phi_{2}}{2}} \frac{\sqrt{\left(\omega_{2} \operatorname{tg} \frac{\phi_{1}}{2}-\omega_{2} \operatorname{tg} \frac{\phi_{1}}{2}\right)\left(\omega_{1} \operatorname{tg} \frac{\phi_{1}}{2}-\omega_{2} \operatorname{tg} \frac{\phi_{2}}{2}\right)}}{\left|\omega_{1}^{2}-\omega_{2}^{2}\right|}
\end{array}
$$

\section{3 whole design and tuning process}

In this part we describe a general step to design and tune a coupler cavity applying this quantitative method.

1. Calculate matching frequency $\omega_{c 0}$ and external quality factor $\mathrm{Q}_{\mathrm{ec} 0}$ of coupler cavity by designed or measured cavity-chain parameter.

2. Calculate the frequency $\omega_{c}$ and external quality factor $\mathrm{Q}_{\mathrm{ec}}$ of model coupler cavity using electromagnetic analyse program, such as MAFIA.

3. Change the size of coupler cavity until $\omega_{c}=\omega_{c 0}, Q_{e c}=Q_{e c 0}$. Record the relationship between $\omega_{c}, Q_{c c}$ and coupler cavity size.

4. Machine actual coupler according to calculation result.

5. Measure frequency $\omega_{c}$ and external quality factor $\mathrm{Q}_{\mathrm{ec}}$ of actual coupler cavity.

6. Fine-tune the coupler cavity according to calculated result.

\section{ONE EXPERIMENTAL TUNING PROCESS}

This section describes one experimental tuning process to verify this quantitative method.

Figure 5 shows the procedure.

(a) Original cavity is undercoupling $(\beta=0.74)$, so the coupling aperture is expanded.

(b) After expanding, the coupling coefficient is increased from 0.74 to 0.85 , but the frequency is still high.

(c) Expanding the radius of coupler cavity, the frequency deviation is decreased from $21 \mathrm{MHz}$ to $6.8 \mathrm{MHz}$, and the coupling coefficient is increased from 0.85 to 0.95 .

(d) Result of slightly expanding the radius eccentrically.

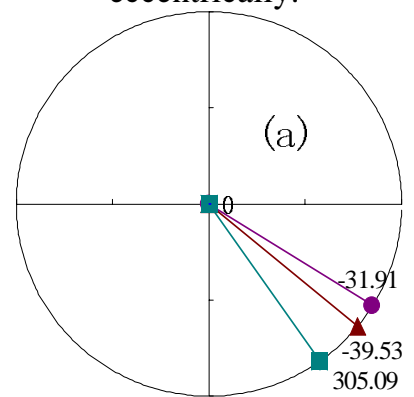

(a) $\beta=0.74, \Delta f=22.58 \mathrm{MHz}$

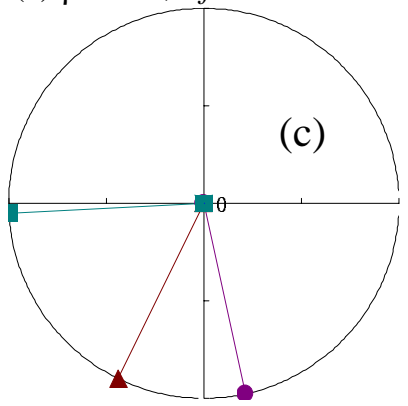

(c) $\beta=0.95, \Delta f=6.80 \mathrm{MHz}$ $\mathrm{f}_{\pi^{\prime} / 2}(2842.42 \mathrm{MHz}) \quad \Delta \mathrm{f}_{\mathrm{m}}(2848.945 \mathrm{MHz}) \quad \mathrm{f}_{\theta 0}(2855.47 \mathrm{MHz})$

Figure 5. Example of one travelling wave accelerator coupler cavity tuning process

\section{CONCLUSION}

The method described here can be used in coupler cavity tuning and simulation. We only need to concern one coupler cavity in tuning and simulation process because matching frequency $\omega_{c 0}$ and external quality factor $\mathrm{Q}_{\mathrm{cc} 0}$ of coupler cavity is calculated at first. So the tuning and simulation process is simplified.

\section{REFERENCES}

[1] E. Westbrook, Microwave Impedance Matching of Feed Waveguides to the Disk-Loaded Accelerator Structure Operating in the $2 \pi / 3$ Mode, SLAC-TN-63103, 1963.

[2] Marie Chanudet, Matching of the Coupler Cavity to Travelling Wave Structures at any Operating Mode, LAL/RT 93-06, 1993 